\title{
Triggering Sublimation-Driven Activity of Main Belt Comets
}

\author{
N. Haghighipour \\ Institute for Astronomy, University of Hawaii-Manoa, Honolulu, HI, 96825, USA \\ nader@ifa.hawaii.edu \\ T. I. Maindl \\ Department of Astrophysics, University of Vienna, Türkenschanzstrasse 17, A-1180 \\ Vienna, Austria \\ C. Schäfer \\ Institut für Astronomie und Astrophysik, Eberhard Karls Universität Tübingen, Auf der \\ Morgenstelle 10, 72076 Tübingen, Germany \\ R. Speith \\ Physikalisches Institut, Eberhard Karls Universität Tübingen, Auf der Morgenstelle 14, \\ 72076 Tübingen, Germany \\ and \\ R. Dvorak \\ Department of Astrophysics, University of Vienna, Türkenschanzstrasse 17, A-1180 \\ Vienna, Austria
}

\begin{abstract}
It has been suggested that the comet-like activity of Main Belt Comets are due to the sublimation of sub-surface water-ice that has been exposed as a result of their surfaces being impacted by m-sized bodies. We have examined the viability of this scenario by simulating impacts between m-sized and km-sized objects using a smooth particle hydrodynamics approach. Simulations have been carried out for different values of the impact velocity and impact angle as well as different target material and water-mass fraction. Results indicate that for the range of impact velocities corresponding to those in the asteroid belt, the depth of an impact crater is slightly larger than $10 \mathrm{~m}$ suggesting that if the activation of MBCs is due to the sublimation of sub-surface water-ice, this ice has to exist no
\end{abstract}


deeper than a few meters from the surface. Results also show that ice-exposure occurs in the bottom and on the interior surface of impact craters as well as the surface of the target where some of the ejected icy inclusions are re-accreted. While our results demonstrate that the impact scenario is indeed a viable mechanism to expose ice and trigger the activity of MBCs, they also indicate that the activity of the current MBCs is likely due to ice sublimation from multiple impact sites and/or the water contents of these objects (and other asteroids in the outer asteroid belt) is larger than the $5 \%$ that is traditionally considered in models of terrestrial planet formation providing more ice for sublimation. We present details of our simulations and discuss their results and implications.

Subject headings: minor planets, asteroids: general — methods: numerical

\section{Introduction}

The detection of comet-like activities in several asteroids during the past decade has generated much interest in the origin of these objects and the mechanism of their activation. Dubbed Main Belt Comets (MBCs), these bodies show dynamical behaviors characteristic of main belt asteroids (e.g., their orbits are entirely in the asteroid belt, some belong to asteroid families, and similar to other main belt asteroids, their Tisserand parameters with respect to Jupiter ${ }^{1}$ are larger than 3 ), whereas unlike most asteroids, they carry tails similar to those of comets. Much of interest in these objects is due to the implication that their comet-like tails and activity are driven by the sublimation of volatile material, presumably water-ice. The latter strongly argues in favor of the idea that water-bearing asteroids, with origins at the outer part of the asteroid belt have been the source of the majority of Earth's water.

At the time of the writing of this article, 8 unambiguous $\mathrm{MBC}^{2} \mathrm{2}^{2}$ were known: $133 \mathrm{P} /(7968)$

\footnotetext{
${ }^{1}$ For a small object, such as an asteroid, that is subject to the gravitational attraction of a central star and the perturbation of a planetary body $\mathrm{P}$, the quantity $a_{\mathrm{P}} / a+2\left[\left(1-e^{2}\right) a / a_{\mathrm{P}}\right]^{1 / 2} \cos i$ is defined as its Tisserand number. In this formula, $a$ is the semimajor axis of the object with respect to the star, $e$ is its orbital eccentricity, $i$ is its orbital inclination, and $a_{\mathrm{P}}$ is the semimajor axis of the planet. In the solar system, the Tisserand number of a small body with respect to Jupiter has, traditionally, been used as a determinant of the cometary or asteroidal nature of its orbit. In general, the Tisserand numbers of comets with respect to Jupiter are smaller than 3, whereas those of asteroids are mostly larger. See also Hsieh \& Haghighipour (2016) for the extent of the viability of using Tisserand number with respect to Jupiter as an orbital determinant.

${ }^{2}$ We call an MBC unambiguous if its activation can only be explained by sublimation of volatiles that
} 
Elst-Pizzaro, 176P/(118401) LINEAR, and 238P/READ (P/2005 U1) (Hsieh \& Jewitt 2006), 259P/Garradd(P/2008 R1) (Jewitt et al. 2009), P/2010 R2 (La Sagra) (Hsieh et al. 2012a), 288P/(300163) P/2006 VW 139 (Hsieh et al. 2012b), P/2012 T1 (PANSTARRS) (Hsieh et al. 2013), and 313P/Gibbs (P/2014 S4) (Hsieh et al. 2015). Table 1 and Figure 1 show the sizes and orbital properties of these objects as well as their locations in the asteroid belt. Among these MBCs, 133P/(7968) Elst-Pizzaro is a member of the 10 Myr old Beagle family $3^{3}$ Nesvorný et al. 2008), and P/2012 T1 (PANSTARRS) and 313P/Gibbs (P/2014 S4) are associated with the $\sim 155$ Myr Lixiaohua family (Hsieh et al. 2013, 2015).

Studies of the dynamical evolution of 133P/(7968) Elst-Pizzaro, 176P/(118401) LINEAR, and 238P/READ (P/2005 U1) have indicated that while the orbits of $133 \mathrm{P} /(7968)$ Elst-Pizzaro and 176P/(118401) LINEAR are stable for 1 Gyr, 238P/READ (P/2005 U1) becomes unstable in about $20 \mathrm{Myr}$ (Haghighipour 2009). Given the association of 133P/(7968) Elst-Pizzaro with the Beagle family, and the proximity of $176 \mathrm{P} /(118401)$ LINEAR, and 238P/READ (P/2005 U1) to the Themis family (Figure 2), these results suggested that 133P/(7968) Elst-Pizzaro, 176P/(118401) LINEAR, and 238P/READ (P/2005 U1) are most likely natives to the asteroid belt and formed in-situ through the breakage of larger asteroidal bodies (Haghighipour 2009, 2010). The orbital instability of 238P/READ (P/2005 U1) can then be attributed to the interaction of this MBC with Jupiter through the 2:1 mean-motion resonance. As shown by Haghighipour (2009), interactions with mean-motion resonances could have affected the dynamics of other MBCs as well causing them to be scattered into orbits away from their birth places. As suggested by this author, the latter implies that, while asteroid families and associations in the outer part of the asteroid belt present promising regions to search for $\mathrm{MBCs}$, stand-alone MBCs can also exist and may be found throughout the asteroid belt. The subsequent detection of MBC 259P/(Garradd)(P/2008 R1) by Jewitt et al. (2009) supported this prediction.

It is important to note that the dynamical analyses presented in Haghighipour (2009) address only the origin of MBCs and not that of their larger parent bodies. While there is no reason to believe that the parent bodies of MBCs could not be native to the asteroid belt, it is also plausible to assume that these objects might have formed in the outer regions of the solar system and were scattered into the asteroid belt during the post-formation evolution of giant planets. Results of the dynamical analysis by Haghighipour (2009), as well as those carried out for other MBCs (Jewitt et al. 2009; Hsieh et al. 2012a b, 2013, 2015), however, suggest that current km-sized MBCs most probably formed in the asteroid belt as a result

have been surfaced as a result of its collision with a small impactor.

${ }^{3}$ Beagle family is a sub-family of the larger Themis family (Nesvorný et al. 2008 . 
of multiple breakage and fragmentation of their parent and grandparent bodies. We refer the reader to Hsieh \& Haghighipour (2016) for more details.

An interesting characteristics of the currently known MBCs is their small sizes. As shown in Table 1, these objects are smaller than $5 \mathrm{~km}$ in size. This small sizes of MBCs has strong implications for the history and mechanism of the activation of these objects. Hsieh et al. (2004) and Hsieh \& Jewitt (2006) studied the mass loss and activation of 133P/(7968) Elst-Pizzaro and showed that, considering an albedo of 0.05 and temperature of $150 \mathrm{~K}$, this $\mathrm{MBC}$ reduces size at a rate of $1 \mathrm{~m} / \mathrm{yr}$. Given that the diameter of 133P/(7968) Elst-Pizzaro is slightly smaller than $4 \mathrm{~km}$, such a rapid rate of size-reduction strongly suggests that the activation of this MBC has been recent. Taking into account the recurrent activity of 133P/(7968) Elst-Pizzaro in 1996, 2002 and 2004, and also observing an increase followed by a decrease in the activity of this MBC during its 2004 perihelion passage, Hsieh et al. (2004) and Hsieh \& Jewitt (2006) suggested that the activity of 133P/(7968) Elst-Pizzaro could not be impact-generated and is probably due to the sublimation of some volatile material, presumably water-ice (an assumption consistent with the location of this MBC in the outer regions of the asteroid belt). These authors suggested that 133P/(7968) Elst-Pizzaro was impacted by a 1-10 m-sized body which excavated its sub-surface ice causing ice to sublimate and create a thin atmosphere and a tail when the orbit of this MBC brings it close to the Sun.

The degree to which the impact of a meter-sized body with a km-sized MBC can excavate sub-surface ice and trigger MBC's activity depends on whether the resulted impact crater will be deep enough to reach the underlying water-ice. The depth of the crater, itself, depends on the impact velocity of the m-sized impactor and the material strength of the MBC. The purpose of this study is to examine the viability of the above-mentioned scenario by simulating the collision of a m-sized body with a km-sized object for different impact velocities and different materials of the impacted body. Specifically, we aim to answer the following three questions:

- What is the maximum depth of an impact crater in a collision between a meter-sized object and a km-sized body for typical impact velocities in the asteroid belt?

- Can such impacts result in the exposure of sub-surface water-ice? In other words, can these impacts differentiate between models of ice-longevity in asteroidal bodies?

- Does the activity of an MBC originate solely from the sublimation of the exposed ice in its crater, or the re-accreted ice will also contribute (and to what extent)?

To answer these questions, we carried out a large number of 3D smooth particle hydro- 
dynamics ( $\mathrm{SPH}$ ) simulations of a collision between a m-sized body and a km-sized object. Simulations were carried out for different values of impact velocities and different values of the material strength and water content of the impacted body.

The outline of this paper is as follows. We begin by describing the details of our model and computational method in section 2. Section 3 has to do with the presentation of our results and their implications for the exposure of sub-surface water-ice and models of icelongevity. We also present in this section a detailed study of the area of ice exposure and its connection to the activation of MBCs. In section 4, we conclude this study by presenting a summary of our findings and their limitations. We also discuss the implications of our results for the water content of asteroidal bodies and their contribution to the Earth's water.

\section{SPH simulations and initial set up}

As mentioned in the introduction, the main purpose of this study is to examine the viability of the impact scenario as a mechanism for exposing water-ice and to differentiate between models of ice-survival in the asteroid belt. For this purpose, and because the depth of an impact crater strongly depends on the material strength of the impacted body, we assume a large range of material strength and consider targets made of a mixture of ice with very hard and very soft materials. For high strength material, we consider basalt and for low strength material, we use tuff. For the sake of comparison, we also consider targets with 100\% ice. Although actual MBCs are most likely carbonaceous chondrites, such a choice of target material will allow us to portray a general picture of the impact scenario, and determine the ranges of size and depth of impact craters for a large range of water content and material strength.

We use a 3D SPH code developed by Schäfer et al. (2007, 2016) and Maindl et al. (2013) to simulate an impact between two bodies. Our code solves the continuity equation and the equation of the conservation of momentum in continuum mechanics. The continuum of the solid body is discretized into mass packages known as SPH particles. The locations of these particles are the sampling points of our numerical method. The SPH particles move as point masses according to the equation of motion. Each particle carries all physical properties (e.g., mass, momentum, energy) of the part of the solid body that it represents. Depending on the type of material, particles may have different material parameters such as bulk and shear modulus, yield strength or different activation thresholds for the development of cracks.

Our code also includes material strength and implements a full elasto-plastic continuum 
mechanics model (see, e.g., Maindl et al. 2013, 2014). To model any specific material, we use the Tillotson equation of state (Tillotson 1962; Melosh 1996) with material parameters as listed in Table 2. To treat fracture and brittle failure, we use the Grady-Kipp fragmentation prescription (Grady \& Kipp 1993; Benz \& Asphaug 1994, 1999). This prescription is based on flaws that are distributed in the material following a Weibull distribution with material-dependent parameters.

When considering basalt or when the target is pure ice, we use the values given by Maindl et al. (2013) for rock and ice parameters in the equation of state and the Weibull distribution. These values are based on measurements by Nakamura et al. (2007) and can be found in their Table 1. Basaltic rock, represented by the parameters of the basalt equation of state, as given by Melosh (1996), is widely used as the material of rocky bodies from cm-sized up to the mantles of large asteroids such as Vesta or Ceres (Melosh \& Tyan |1997; Agnor \& Asphaug 2004; Nakamura \& Michel 2009; Michel 2009). We use slightly modified parameters similar to those used by Benz \& Asphaug (1999), and apply a tensorial

correction as suggested by Schäfer et al. (2007, 2016) to ensure first-order consistency. When simulating collisions, we implement our damage model using Weibull parameters $m$ and $k$. For basalt, we use $m=16$ and $k=10^{61}\left(\mathrm{~m}^{-3}\right)$ (Nakamura et al. 2007) and for water-ice, we use $m=9.1$ and $k=10^{46}\left(\mathrm{~m}^{-3}\right)$ (Lange et al. 1984).

When considering tuff, because there are no data on the flaw distribution in this material, and also because tuff is not as brittle as ice or basalt, we do not model fractures. Instead, we assume ductile deformation and fragmentation.

To simulate collisions, we resolve the combined system of the impactor and target into approximately 500,000 SPH particles. Following Maindl et al. (2014) and because the impact timescales are very short compared to the time of the influence of the gravitational force of the target body (the collision velocities are in the order of $\mathrm{km} / \mathrm{s}$ whereas the MBCs' surface escape velocities are less than a few $\mathrm{m} / \mathrm{s}$, see Table 1), we simulate collisions without self-gravity. To analyze the evolution of the system during each impact, we take 250-500 snapshots every $0.4 \mathrm{~ms}$. In between the snapshots, time integration is continued with an adaptive step-size.

\section{Impact simulations}

We simulated collisions between a m-sized impactor and a km-sized body with the target being a mix of basalt and ice, tuff and ice, or pure water-ice. When using basalt and tuff, we considered the water-mass fraction of the target to be $0 \%, 5 \%$ (following the conventional 
consideration of the water-mass fraction of asteroids in models of terrestrial planet formation as in e.g., Raymond et al. 2004, 2009; Izidoro et al. 2013, 2014; Haghighipour \& Winter (2016), and 50\%. Because the size and depth of an impact crater varies with the energy imparted to the target, we carried out simulations for different values of impact velocity and impact angle.

The fact that the activation of MBCs is recent indicates that irrespective of the origin of these objects, collisions between impactors and MBCs must have happened when the projectile and target were both in the asteroid belt. As shown by Bottke et al. (1994), the mean impact velocity of bodies in the asteroid belt is $\sim 5.3 \mathrm{~km} \mathrm{~s}^{-1}$ with the most probable value being around $4.4 \mathrm{~km} \mathrm{~s}^{-1}$ for bodies larger than $50 \mathrm{~km}$. This indicates that the collision velocity distribution, while having a long high-velocity tail is heavier towards smaller velocities. We assume an abundance of objects significantly smaller than $50 \mathrm{~km}$ on similar orbits (i.e., comparable semi-major axes, eccentricities and inclinations) with $e \lesssim 0.25$, inside the asteroid belt. Given their similar orbits and in the absence of significant mutual gravitational interaction, collisions between these bodies will occur with velocities much smaller than several $\mathrm{km} \mathrm{s}^{-1}$. Combining this assumption with the finding of Bottke et al. (1994), we therefore considered impact velocities in the range of $0.5 \mathrm{~km} \mathrm{~s}^{-1}$ to $5.3 \mathrm{~km} \mathrm{~s}^{-1}$, and added an additional tail to our velocity distribution towards smaller values extending down to $100 \mathrm{~m} \mathrm{~s}^{-1}$. We also chose the impact angle $(\alpha)$ to vary in the range of 0 (head-on collision) to $60^{\circ}$.

Figure 3 shows snapshots of a sample of our simulations. The target is made of basalt and has $5 \%$ water in mass. The impact velocity is $4.4 \mathrm{~km} \mathrm{~s}^{-1}$ and the impact angles are 0 and $30^{\circ}$. To reduce the amount of unnecessary computations, we limited the region of simulation in the target to a volume of $30 \mathrm{~m} \times 30 \mathrm{~m} \times 30 \mathrm{~m}$. Figure 4 shows the final state of head-on $(\alpha=0)$ impacts for tuff and basaltic targets with water-mass fractions of $0 \%, 5 \%$ and $50 \%$. As shown in these figures, ice particles are exposed on the bottom and walls of the crater, and the depth and morphology of the impact crater vary with the type of the target material and its water content. In the next section, we present an analysis of these results and discuss their implications for the models of ice-longevity in asteroids and the activation of MBCs.

Figures 3 and 4 also show that the debris generated after the impact contains both ice and rock. While some of these ice particles and rock debris leave the entire impacted body, some are re-accreted and settle on the surface of the target. We discuss this process and its contribution to the activation of MBCs later in this section. We note that we consider the entire energy of impact to be imparted to the target and do not consider evaporation of ice particles due to the heat of the impact. We allow shocks to propagate throughout 
the target and analyze the target's response using our damage model as described in the

previous section. Movies of some of our impact simulations can be downloaded from the electronic supplementary material.

\subsection{Calculation and analysis of crater depth}

Theoretically, the depth of a crater is given by the distance of its deepest point to the top of the crater. In the context of our SPH simulations, this implies that to determine the depth of a crater, we need to identify the crater's deepest SPH particle. However, because in our simulations, impact velocities are high, craters form in a small fraction of a second after an impact. This, combined with the negligible gravity of the target results in the appearance of spurious SPH particles. The latter makes the calculation of the depth of a crater based on the locations of individual SPH particles, unreliable.

To avoid this complication, we measure the depth of a crater using an indirect method. We begin by considering a cross section of the crater in the direction of the vertical component of the impact velocity and passing through the crater's plane of symmetry. In this cross section, we fit an ellipse to the crater. To ensure that our measurement of the depth of the crater includes a sufficient number of SPH particles at the bottom of the crater, we also consider a width for this fitted ellipse in the direction perpendicular to the above cross section (i.e., in the direction parallel to the horizontal component of the impact velocity). This width is chosen such that while the fitted elliptical band contains sufficiently large number of particles to encompass the bottom of the crater, its particle number will also be small enough to avoid obscuring the crater's shape. As mentioned above, impact craters form in a fraction of a second. To ensure that prior to the fitting, post-impact variations in the shape and depth of the crater have become negligibly small, we allow a time lapse of $\Delta t$. This quantity is chosen such that it is as large as necessary to get the final shape of the crater and as small as possible to avoid the shock waves of the impact to hit the boundary before the crater forms. Next, while ignoring single, scattered SPH particles, we determine the location of the bottom of the elliptical band with deepest penetration into the target. We define the crater depth to be the distance between this deepest point to the surface of the target. Figure 5 illustrates this methodology for an impact velocity of $4.4 \mathrm{~km} \mathrm{~s}^{-1}$ at a $30^{\circ}$ angle onto a basaltic target with $5 \%$ water-ice. As shown here, the crater has a depth of $3.39 \mathrm{~m}$. Uncertainties in calculating the depth of a crater in this method depend on the smoothing length of the simulations which varies by the size of the target in each individual impact. We estimate the errors to be in the range of $0.2-0.5 \mathrm{~m}$. 


\subsection{Implications for models of ice-longevity in asteroids}

In order for water-ice to be exposed, the impact crater has to be deep enough to reach the MBC's internal ice. While the depth of an impact crater strongly depends on the impact velocity, the depth where the ice is buried depends on the physical and dynamical properties of the MBC. As suggested by Schörghofer (2008), in asteroids with mean surface temperatures smaller than $145 \mathrm{~K}$ (e.g., the MBC 133P/(7968) Elst-Pizarro), a layer of only a few tens of centimeter of dust would be able to prevent the ice from sublimating and would allow the asteroid to maintain its internal ice for the age of the solar system. According to this model, an impact crater of only a few meters in depth would be sufficient to expose

sub-surface water-ice. A different model by Prialnik \& Rosenberg (2009), however, makes drastically different predictions. These authors suggest that an MBC such as 133P/(7968) Elst-Pizarro can maintain only crystalline water-ice and only in depths ranging from $50 \mathrm{~m}$ to $150 \mathrm{~m}$.

We use the results of our impact simulations to differentiate between these two models. Figure 6 shows crater depths in all our simulations in terms of the vertical component of the impact velocity. As expected, the depth of a crater strongly depends on the material strength of the target. For a given value of the vertical component of the impact velocity, the crater depth increases by varying target material from basalt to tuff, and more importantly, by increasing target's water-mass fraction. An interesting results shown in figure 6 is that even when the target is considered to be pure ice, the crater depth does not exceed $12 \mathrm{~m}$. For the conventional case of $5 \%$ water-mass fraction, the depth of the crater stays below $6 \mathrm{~m}$ in all simulations. Our results suggest that if the activation of MBCs is due to the sublimation of water-ice, the ice must be buried in the top few meters. We note that the uncertainties due to the SPH method are approximately $0.5-0.9 \mathrm{~m}$.

\subsection{Implications for the area of ice exposure}

\subsubsection{Interior Surface of the Impact Crater}

An inspection of figures 3 and 4 indicates that in addition to the bottom of a crater, ice may also be exposed on the crater's interior surface. This suggests that although during an impact, some of the target material, including its ice is lost due to vaporization and scattering to the space and regions outside the crater, a considerable amount of ice may still remain on the inner surface of the crater and contribute to the activation of the MBC. To

calculate the interior area of an impact crater, we fit the crater with a spheroid and use the fitted spheroid to determine the crater's diameter. Knowing the crater depth as explained 
in the previous section, we calculate the interior area of a crater by calculating the surface area of the fitted spheroid. Figure 7 shows the results for all our head-on collisions. As expected, the interior area of an impact crater increases with increasing the target's water content. That is due to the fact that, as shown by the columns on material moduli in Table 2 , while basalt shows much resistance to deformation, water-ice is significantly less resistant to compression, shear, and ductile deformation/yield. As a result, a material being a blend of basalt and water-ice will resist an impact to a lesser extent if its water-ice fraction becomes higher. This in turn translates into larger craters with increasing water content.

Figure 7 shows that despite the fact that tuff is even weaker than water-ice with respect to deformation after an impact, adding ice inclusions into tuff also results in larger craters. The reason can be attributed to tuff being a non-brittle, ductile material that maintains its ductile state and does not become cohesionless and liquid-like when it gets damaged by an impact. As shown in figure 7, this effect gets more pronounced for higher impact energies that result in higher degrees of damage to the water ice.

Assuming that the water-ice is present under a thin layer of surface material, the exposed water-ice on the crater walls of an $\mathrm{MBC}$ will have significant contribution to its activity. Table 3 lists the surface areas of the active regions of the currently known MBCs. The footnote at the bottom of this table compares the recent data with other observation-based estimates to illustrate an expected order-of-magnitude certainty-level. Our simulations yield crater surfaces of up to a few hundred square meters which, compared to the values in Table 3, are too small, by 1-2 orders of magnitude, to account for the observed activation. If activity is to be explained by impact craters, these results suggest that multiple impact sites may be active and/or the water content of the object may be larger than the anticipated

$5 \%$ so that crater sizes are larger and expose more water-ice. Also, as explained in the next section, possible re-accreted ejected material can provide an additional mechanism for supplying sublimating water-ice after an impact.

\subsubsection{Area of Ice-Exposure on the surface of the $M B C$}

The area of ice-exposure is not limited to only the bottom and interior surface of an impact crater. Parts of the material ejected out of a crater may be re-accreted on the surface of the target and form an area where ice may be exposed. The amount of ejected material, its water content, and the amount of re-accretion strongly depend on the impact velocity as well as the material strength and gravity of the target. To determine the mass of the reaccreted ice and its exposed area, we followed the dynamical evolution of the scattered ejecta by identifying fragments via a friend-of-friends algorithm with the SPH smoothing length as 
the discriminating criterion. Our analysis indicated that, because of the low escape velocities of objects with sizes similar to those of the currently known MBCs, most of the fragments that are generated in high velocity impacts are scattered out of the system. Only when the impact velocities are low and the target has a high water content, some of the fragments will have low enough velocities to be re-accreted. Figure 8 shows the amount of the ejected mass in terms of the velocities of the ejecta for impact velocities between $100 \mathrm{~m} \mathrm{~s}^{-1}$ and $1000 \mathrm{~m}$ $\mathrm{s}^{-1}$ and an impact angle of $30^{\circ}$. The impactor was m-sized. The target was taken to be a mix of basalt and ice with $0 \%$ and $50 \%$ water content. For the sake of comparison, a pure-ice target was also considered. As shown here, most of the scattered material is ejected with velocities larger than $5 \mathrm{~m} \mathrm{~s}^{-1}$. This velocity is a few $\mathrm{m} \mathrm{s}^{-1}$ larger than the escape velocity of a typical MBC which is smaller than $2.5 \mathrm{~m} \mathrm{~s}^{-1}$ (see Table 1). Considering $2.5 \mathrm{~m} \mathrm{~s}^{-1}$ as the uppermost limit of an MBC's escape velocity, figure 8 indicates that only a small amount of the ejected material may be re-accreted by the target. It is expected that slower impacts onto targets with higher water contents yield more re-accreted material.

To demonstrate the implications of these results, we assume, merely for the sake of argument, that MBC 133P/(7968) Elst-Pizzaro is made of a mix of basalt and water-ice, and has been subject to the impact scenario of figure 8. Figure 9 shows the amount of the re-accreted mass on the surface of this (hypothetical) MBC, after an impact, in terms of the impact velocity and for two values of water-mass ratio of 0 and $50 \%$. The case of a pure-ice $\mathrm{MBC}$ is also shown for a comparison. As shown here, an increase of the impact velocity from $100 \mathrm{~m} \mathrm{~s}^{-1}$ to $500 \mathrm{~m} \mathrm{~s}^{-1}$ decreases the amount of re-accreted material by an order of magnitude. While an accreted mass of $10^{4} \mathrm{~kg}$ for an impact velocity of $100 \mathrm{~m} \mathrm{~s}^{-1}$, combined with the exposed ice at the bottom and interior area of the impact crater may be enough to ignite the activity of 133P/(7968) Elst-Pizzaro, other MBCs such as 259P/Garradd (P/2008 R1) and P/2010 R2 (La Sagra) that have smaller escape velocities will not be able to accrete as much material. The fact that these and other MBCs do show activities suggests that MBCs may contain more water than considered here and/or their activities may be due to ice-sublimation in several active craters resulted from multiple impacts at low velocities.

\section{Summary and Concluding Remarks}

We carried out an extensive study of the impact of a m-sized body with a km-sized object to examine the viability of the impact scenario as a mechanism to expose embedded waterice and trigger the activation of main belt comets. We simulated impact events considering different water-mass fractions for very hard and very soft targets. We showed that ice can be exposed on the bottom and interior surface of an impact crater and may also be re-accreted 
on the surface of an MBC, creating a large combined area of ice sublimation. Results of our simulations indicated that

- considering the current range of impact velocities in the asteroid belt, the depth of an impact crater is only slightly larger than $10 \mathrm{~m}$ even when the target is assumed to be of the softest material. This suggests that if the activation of MBCs is due to the sublimation of water-ice, ice must be buried in the top few meters of the object. The latter is consistent with the model by Schörghofer (2008) who showed that a layer of dust with a thickness of only a few tens of cm can prevent water-ice in asteroids from sublimating for the age of the solar system;

- substantial amount of ice may be exposed on the inner surface of an impact crater contributing to the activation of an MBC. The size and therefore the inner area of a crater is larger for targets with higher water-mass fraction;

- some of the ejected material may be re-accreted when the impact velocities are low (e.g., $\lesssim 100 \mathrm{~m} / \mathrm{s}$ ), and may contribute to the activation of MBCs. Calculations by Hsieh et al. (2004) and Hsieh \& Jewitt (2006) suggested a very large ice-exposure area to account for the activity of MBC 133P/(7968) Elst-Pizzaro. Our simulations indicated that while for an object similar to this $\mathrm{MBC}$, the exposed ices on the bottom and interior surface of an impact crater, combined with the ice re-accreted on the surface of the object may be large enough to account for its activation, in general, due to the small gravity of MBCs, the amount of re-accreted material on the surfaces of these objects is too small to contribute to their activity. The latter implies that the activity of MBCs is most likely due to ice-sublimation from multiple impact craters and/or the water content of these bodies are larger than the anticipated $5 \%$, providing more ice in exposed areas for sublimation.

Although the simulations presented here portray the impact scenario as a viable mechanism for exposing sub-surface ice to ignite the activity of MBCs, they contain some limiting assumptions. First, we did not consider porosity for our targets. Including porosity is a complicated task that requires developing complex mathematical models. The latter is currently under development and we expect the results to be ready for publication in not so distant future. However, given the simulations presented here, we do not expect the result to change drastically even when porosity is included.

We considered the target material to be basalt or tuff. However, MBCs are most likely made of carbonaceous chondrites. That means, in order to simulate impact events, the knowledge of the equation of state of this material as well as the flaw distribution for the 
fracture model are required, which are unknown. Our approach, that is, simulating impacts using a very hard (basalt) and a very soft (tuff) target allows us to portray a general picture of the impact scenario as a mechanism for ice exposure that can be applied to a variety of targets with material strengths between these two ranges.

We believe that our SPH model of elasto-plastic continuum mechanics with brittle failure for applicable materials covers a valid range of target substances, even though for most waterice weight fractions, the bulk densities of our materials (see Table 2) differ from known densities of MBCs of about $1300 \mathrm{~kg} \mathrm{~m}^{-3}$ to $1400 \mathrm{~kg} \mathrm{~m}^{-3}$ (Hsieh et al. 2004; Hsieh \& Jewitt |2006; Jewitt et al. 2009; Hsieh et al. 2011; Hsieh 2014; Hsieh et al. 2015). Also, our damage and plasticity models account for reduced material strength and cohesion when under load, for instance by an impact, down to entirely cohesionless material at the impact site or during a break-up (Hirabayashi et al. 2014).

Our simulations did not account for the vaporization of ice inclusions due to the heat of an impact. It is expected that after an impact, some of the embedded ice will vaporize and leave the target. Although some of this vaporized ice may also condense and settle on the surface of the target, when put in the context of the activity of the currently known MBCs, the loss of ice due to vaporization implies that a single impact on a small, km-sized MBC may not be able to expose sufficient amount of ice to account for its activity. The latter suggests that the activity of MBCs is likely due to ice sublimation from multiple impact sites, and/or the water content of MBCs (and other asteroids in the outer part of the asteroid belt, for that matter) may be higher than the conventional $5 \%$ that is traditionally considered in models of terrestrial planet formation to provide more ice in the exposed areas.

Finally, we would like to note that we did not consider a regolith layer on the top of a target's icy material. We assumed ice to exist everywhere and considered a random distribution of icy inclusions throughout the target. While it is expected that the inclusion of a regolith layer would decrease the depth of an impact crater, the micro-impacts created by the scattered fragments of this layer may shatter the surface of the target in other regions to the extent that the underlying ice may be exposed on a larger area. The latter is currently being studied.

We would like to thank the referee for critically reading our paper and for his/her valuable comments that improved our manuscript. NH acknowledges support from NASA PAST program under grant NNX14AJ38G. TIM and RD acknowledge support from FWF Austrian Science Funds under projects S11603-N16. 


\section{REFERENCES}

Agnor, C. \& Asphaug, E. 2004, ApJ, 613, L157

Benz, W. \& Asphaug, E. 1994, Icarus, 107, 98

Benz, W. \& Asphaug, E. 1999, Icarus, 142, 5

Bottke, W. F., Nolan, M. C., Greenberg, R. \& Kovoord, R. A. 1994, Icarus, 107, 255

Grady, D. E., \& Kipp, M. E. 1993, in: J. R. Asay \& M. Shahinpoor (Eds), High Pressure Shock Compression of Solids (Springer-Verlag, New York), Chapter 5, 265

Haghighipour, N. 2009, Meteor. \& Planet. Sci., 44, 1863

Haghighipour, N. 2010, in: Icy Bodies of the Solar System, Proceedings of the IAU Symposium 263, 207

Haghighipour, N. \& Winter, O. C. 2016, Celest. Mech. Dynamic. Astron., 124, 235

Heap, M. J., Baud, P., Meredith, P. G., Vinciguerra, S. \& Reuschlé, T. 2014, Solid Earth, 5,25

Hirabayashi, M., Scheeres, D. J., Sánchez, D. P., \& Gabriel, T. 2014, ApJ, 789, L12

Hsieh, H. H., Jewitt, D. C., \& Fernández, Y. R. 2004, AJ, 127, 2997

Hsieh, H. H. \& Jewitt, D. 2006, Science, 312, 561

Hsieh, H. H., Meech, K. J., \& Pittichová, J. 2011, ApJ, 736, L18

Hsieh H. H., et al. 2012a, AJ, 143, 104

Hsieh H. H., et al. 2012b, ApJ, 748, L15

Hsieh H. H., et al. 2013, ApJ,771, L1

Hsieh, H. H. 2014, Icarus, 243, 16

Hsieh H. H., et al. 2015, ApJ, 800, L16

Hsieh, H. H. \& Haghighipour, N. 2016, Icarus, in press

Izidoro, A., de Souza Torres, K., Winter, O. C. \& Haghighipour, N. 2013, ApJ, 767, 54

Izidoro, A., Haghighipour, N., Winter, O. C ., Tsuchida, M. 2014, ApJ, 782, 31 
Jewitt, D., Yang, B., Haghighipour, N. 2009, ApJ, 137, 4313

Jewitt, D., Hsieh, H. \& Agarwal, J. 2015, in P. Michel, F. DeMeo \& W. Bottke (Eds), ASTEROIDS IV (University of Arizona Press, Tucson), 895, 221

Lange, M. A., Ahrens, T. J. \& Boslough, M. B. 1984, Icarus, 58, 383

Maindl, T. I., Schäfer, C., Speith, R., Süli, Á., Forgács-Dajka, E., \& Dvorak, R. 2013, A\&A, 334,996

Maindl, T. I., Dvorak, R., Schäfer, C., \& Speith, R. 2014 , in: Knežević, Z. \& Lemaître, A. (Eds), Complex Planetary Systems, Proceedings of the IAU Symposium 310, 138

Melosh, H. J. 1996, Impact Cratering, Oxford University Press

Melosh, H. J. \& Ryan, E. V. 1997, Icarus, 129, 562

Michel, P. 2009, Lect. Notes Phys., 758, 99

Nakamura, A. M., Michel, P. \& Setoh, M. 2007, JGRE, 112, CiteID E02001

Nakamura, A. M. \& Michel, P. 2009, Lect. Notes Phys., 758, 71

Nesvorný, D., Bottke, W. F., Vokrouhlický, D., et al. 2008, ApJ, 679, 143

Prialnik, D. \& Rosenberg, E. D. 2009, MNRAS, 399, L79

Raymond, S. N., Quinn, T. \& Lunine, J. I. 2004, Icarus, 168, 1

Raymond, S. N., O’Brien, D. P., Morbidelli, A. \& Kaib, N. A., 2009, Icarus, 203, 644

Schäfer, C., Speith, R., \& Kley, W. 2007, A\&A, 325, 84

Schäfer, C., Riecker, S., Maindl, T. I., Scherrer, S., Speith, R., \& Kley, W. 2016, A\&A, 590, A19

Schorghofer, N. 2008, ApJ, 682, 697

Stowe, R. L. 1969, Tech. Rep. C-69-1, U.S. Army Engineering Waterways Experiment Station, Vicksburg, Miss.

Tillotson, J. H. 1962. Metallic Equations of State for Hyper-velocity Impact. General Atomic Report GA-3216, General Atomic, San Diego, CA. 


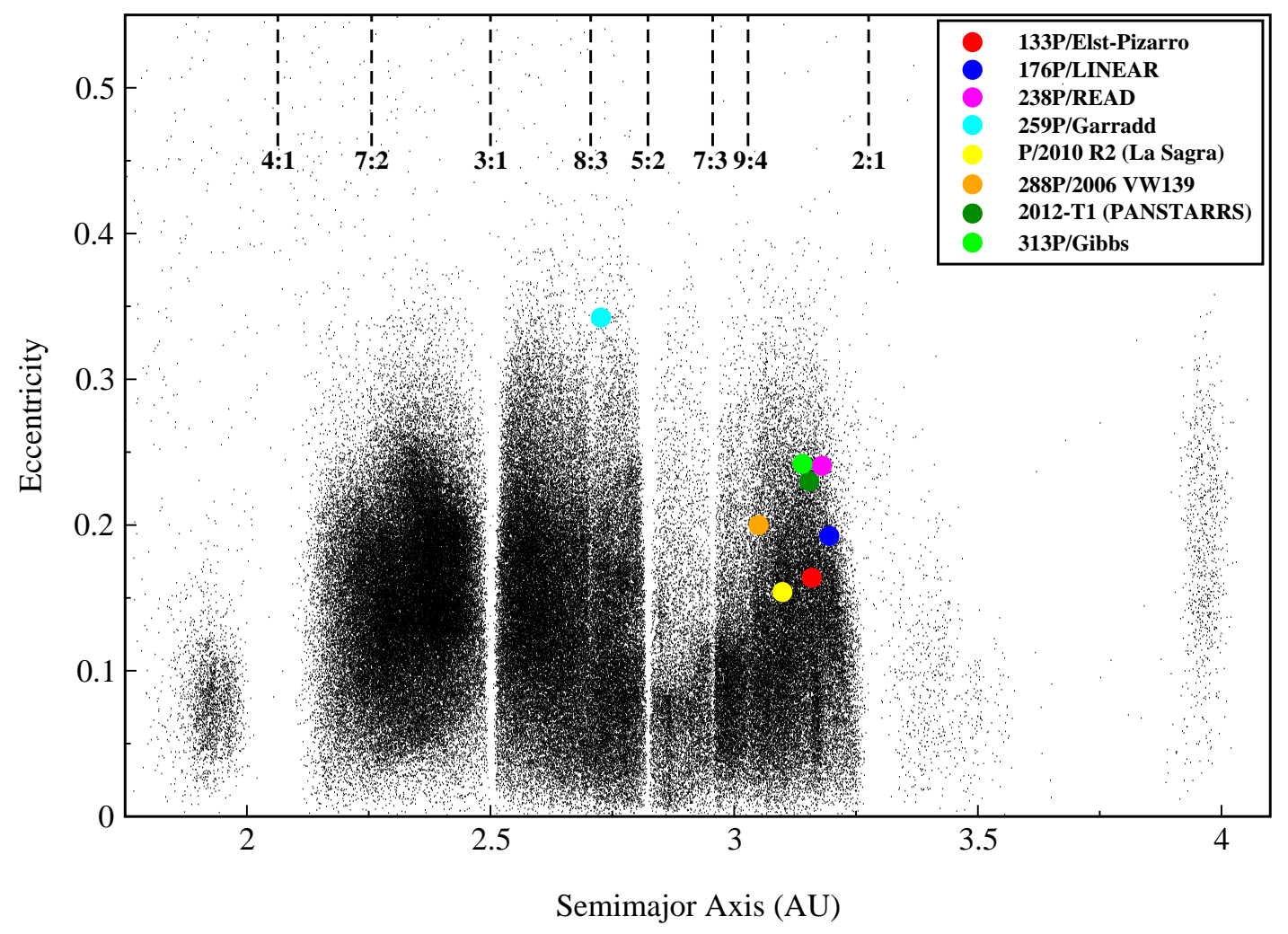

Fig. 1.- Locations of the currently known MBCs in the asteroid belt. The background shows all asteroids and the positions of mean-motion resonances with Jupiter. 


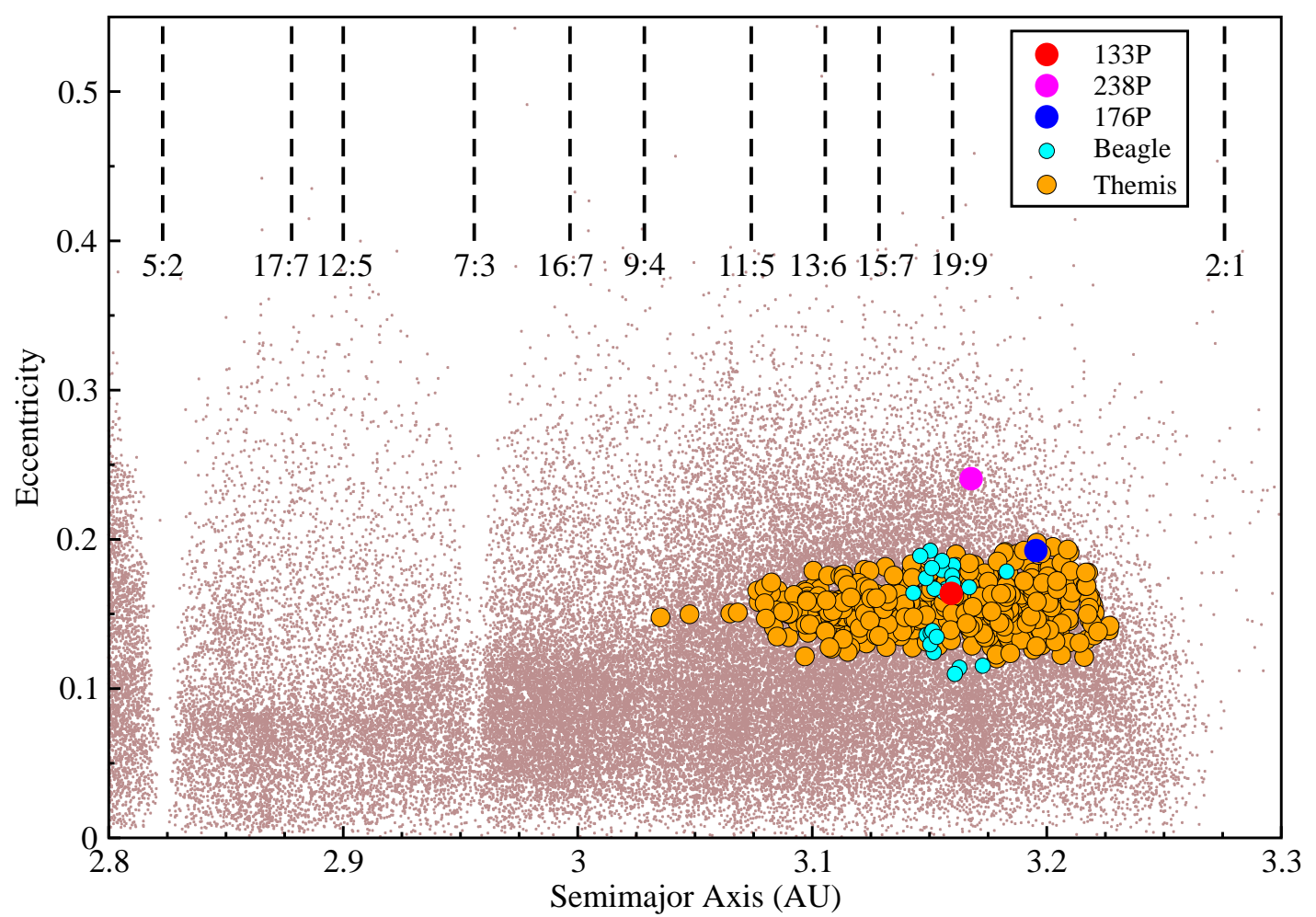

Fig. 2.- Association of MBCs 133P/(7968) Elst-Pizzaro, 176P/(118401) LINEAR, and 238P/READ (P/2005 U1) with the Themis and Beagle families. The background shows a portion of the asteroid belt that is in the vicinity of the Themis family and the locations of mean-motion resonances with Jupiter. 

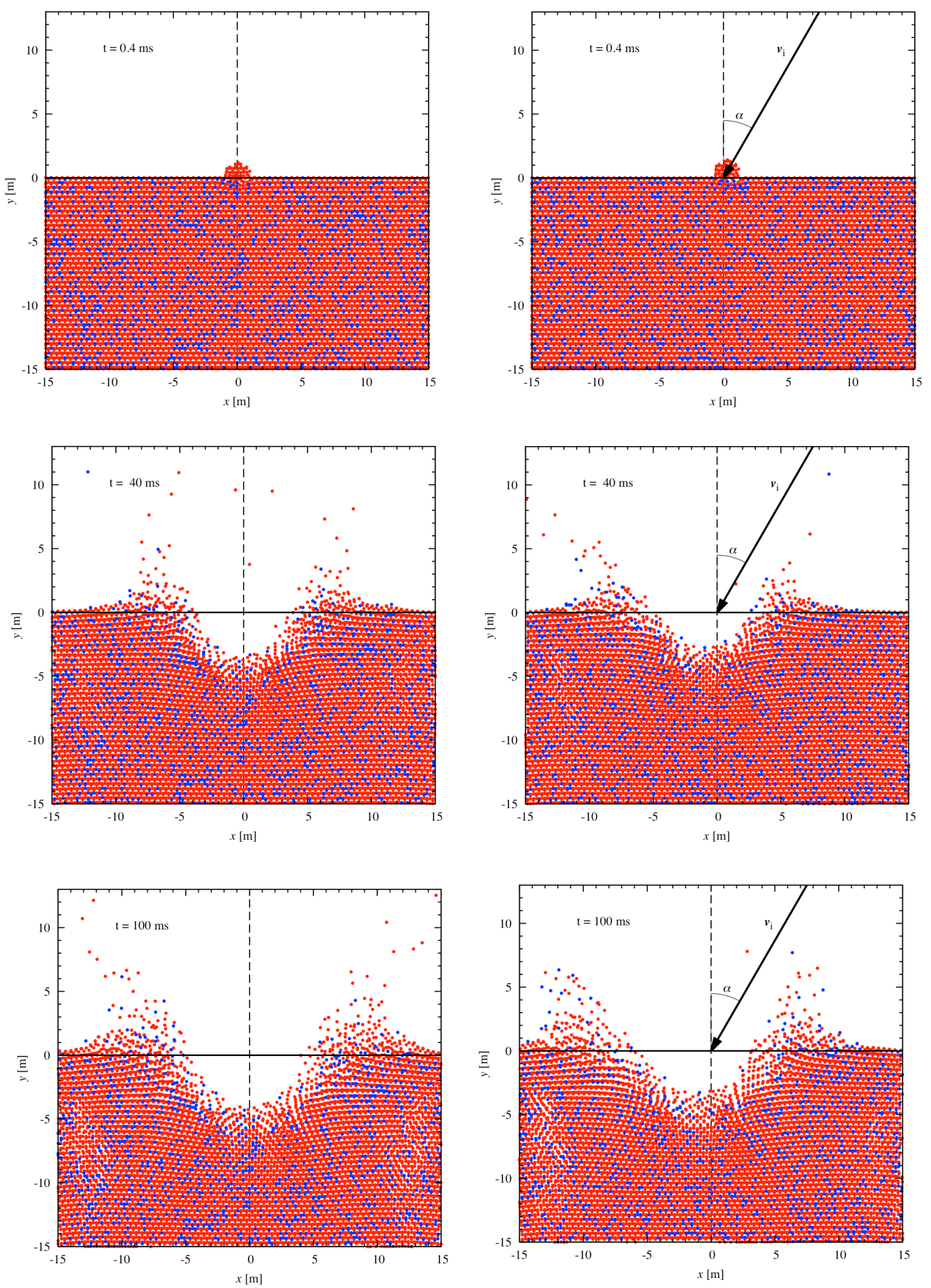

Fig. 3.- Snapshots of the collision of a m-sized object with a basaltic target with a $5 \%$ water-mass fraction. The impactor is made of pure basalt. The impact velocity is $4.4 \mathrm{~km}$ $\mathrm{s}^{-1}$ and $\alpha=0$ (left) and $30^{\circ}$ (right). As shown here, after the impact, ice is exposed at the bottom and on the interior surface of the crater. 

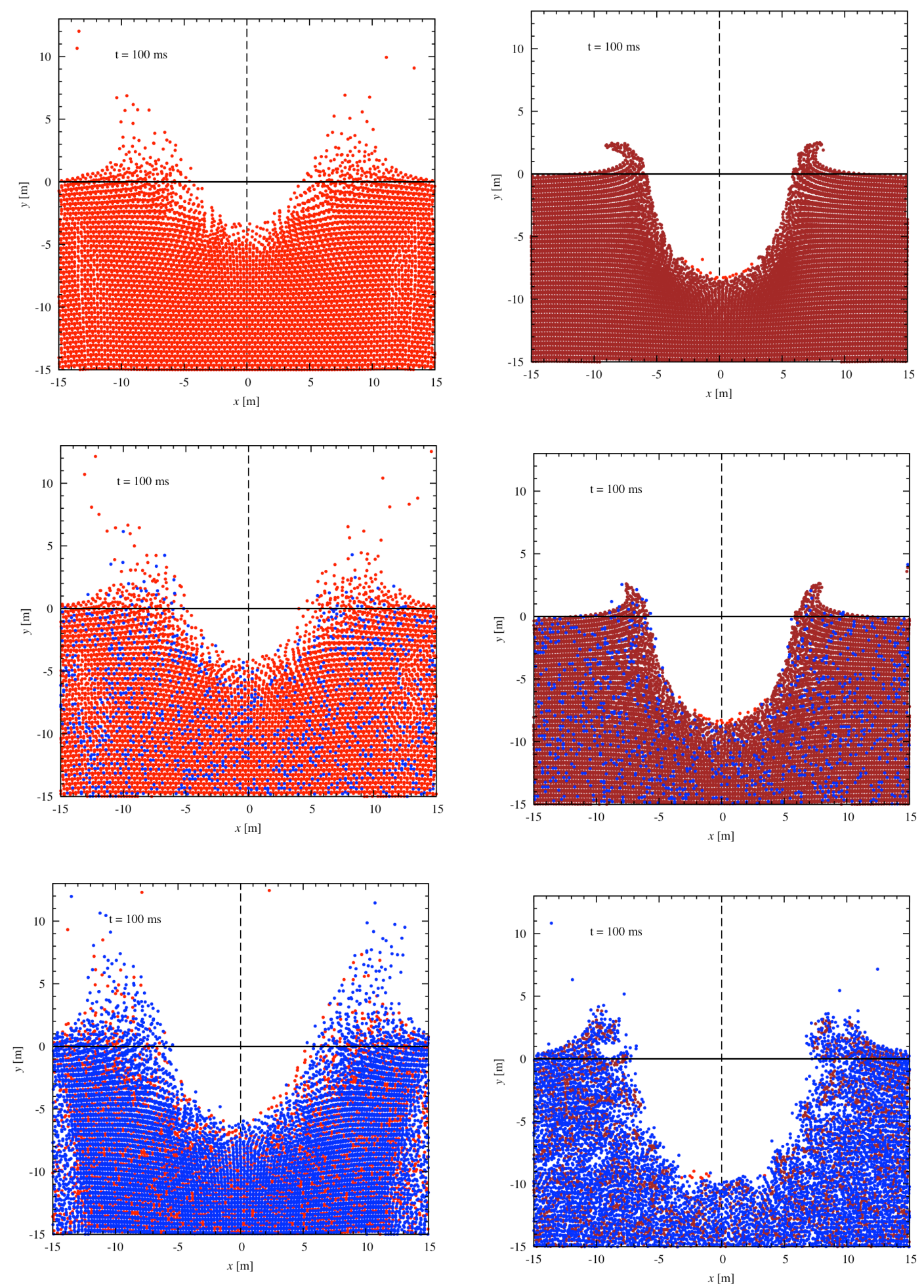

Fig. 4. - State of the impact crater in a $4.4 \mathrm{~km} \mathrm{~s}^{-1}$ head-on collision (as in figure 3) $100 \mathrm{~ms}$ after the impact. The target on the left column is made of basalt and the one on the right is made of tuff. From top to bottom, the water-mass fraction of the target is 0, 5\% and 50\%, respectively. 

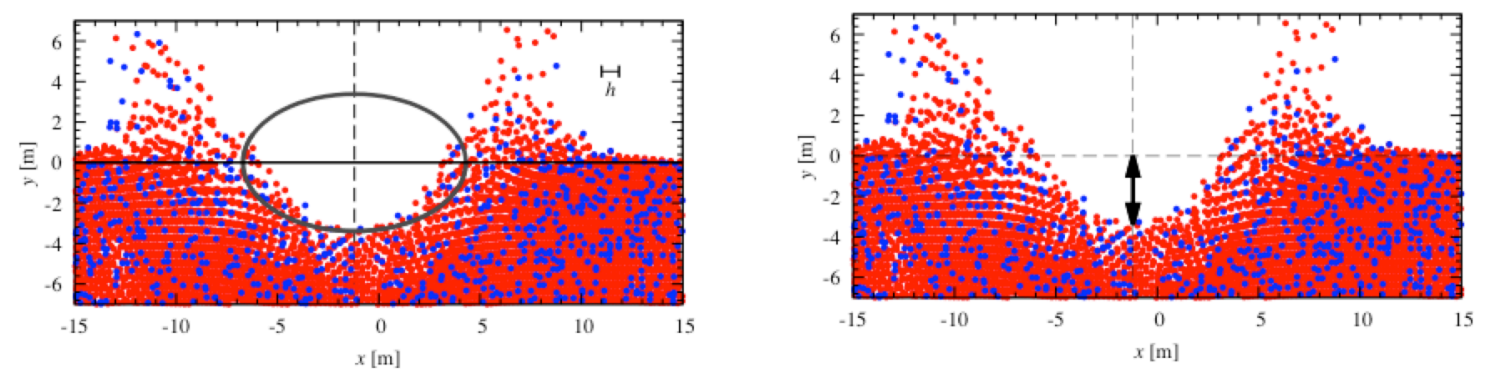

Fig. 5.- Determining the depth of an impact crater. The target is made of basalt with a $5 \%$ water-mass fraction. The impact velocity is $4.4 \mathrm{~km} \mathrm{~s}^{-1}$ and the impact angle is $30^{\circ}$. The panel on the left shows the fitted ellipse in the the cross section of the crater along the vertical component of the impact velocity (the $x$-component). The fitted ellipse is slightly extended along the $z$-axis, perpendicular to the page. The panel on the right shows the depth of the crater after identifying the lowest point of the fitted elliptical band at $3.39 \mathrm{~m}$. 


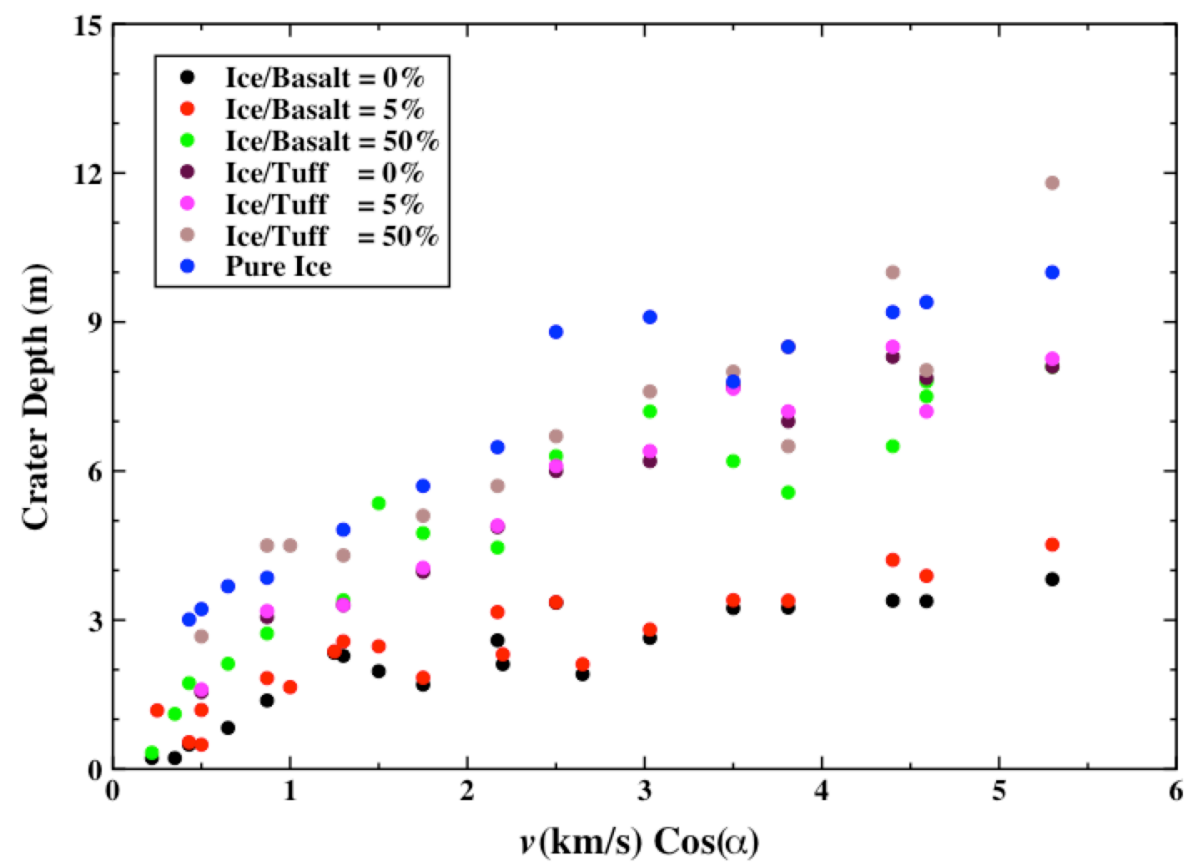

Fig. 6.- Variations of crater depth in terms of the magnitude of the vertical component of the impact velocity and the water content of the target for basalt, tuff and pure ice. As shown here, the depth of the crater increases by increasing the target's water-mass fraction. However, it does not exceed $12 \mathrm{~m}$. 


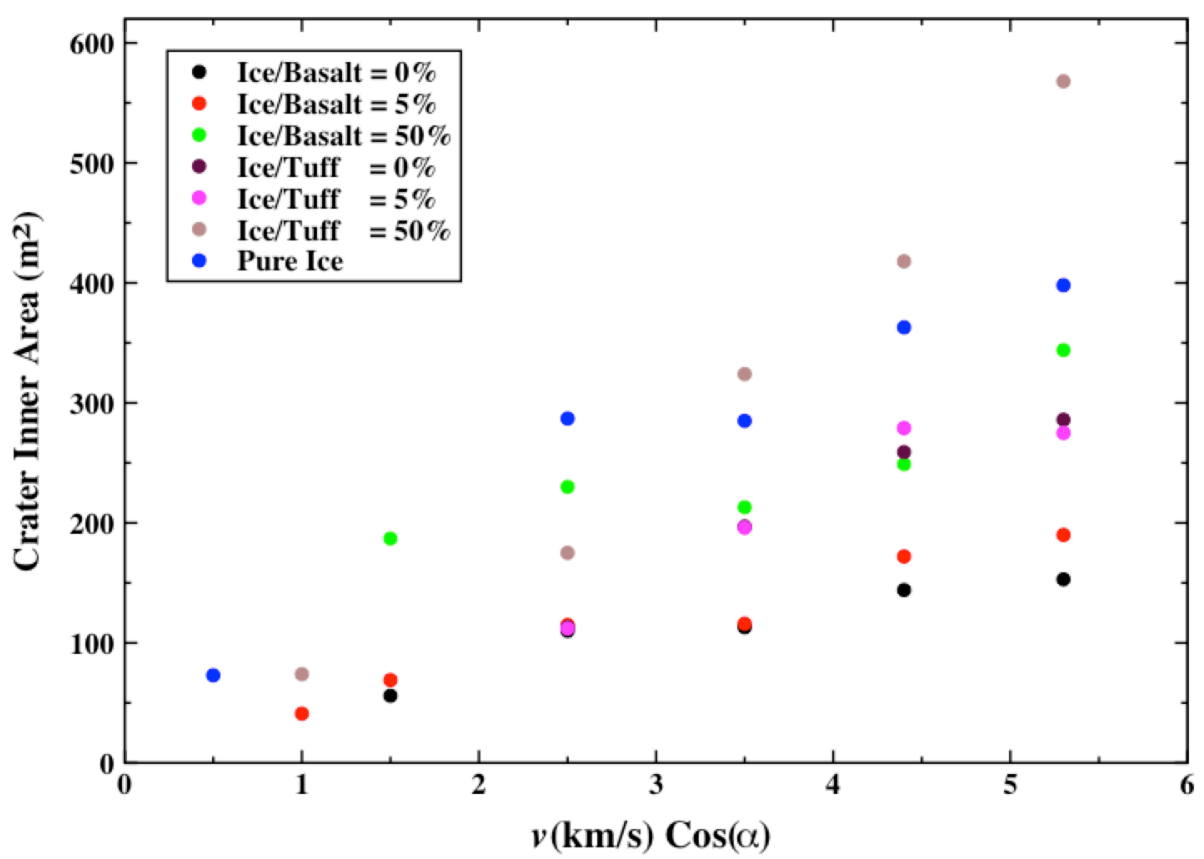

Fig. 7.- Variations of the inner area of an impact crater in terms of the impact velocity in a head-on collision. The target is made of basalt or tuff with different water-mass fractions, or it is pure ice. As shown here, the inner area of a crater increases by increasing the target's water content. 

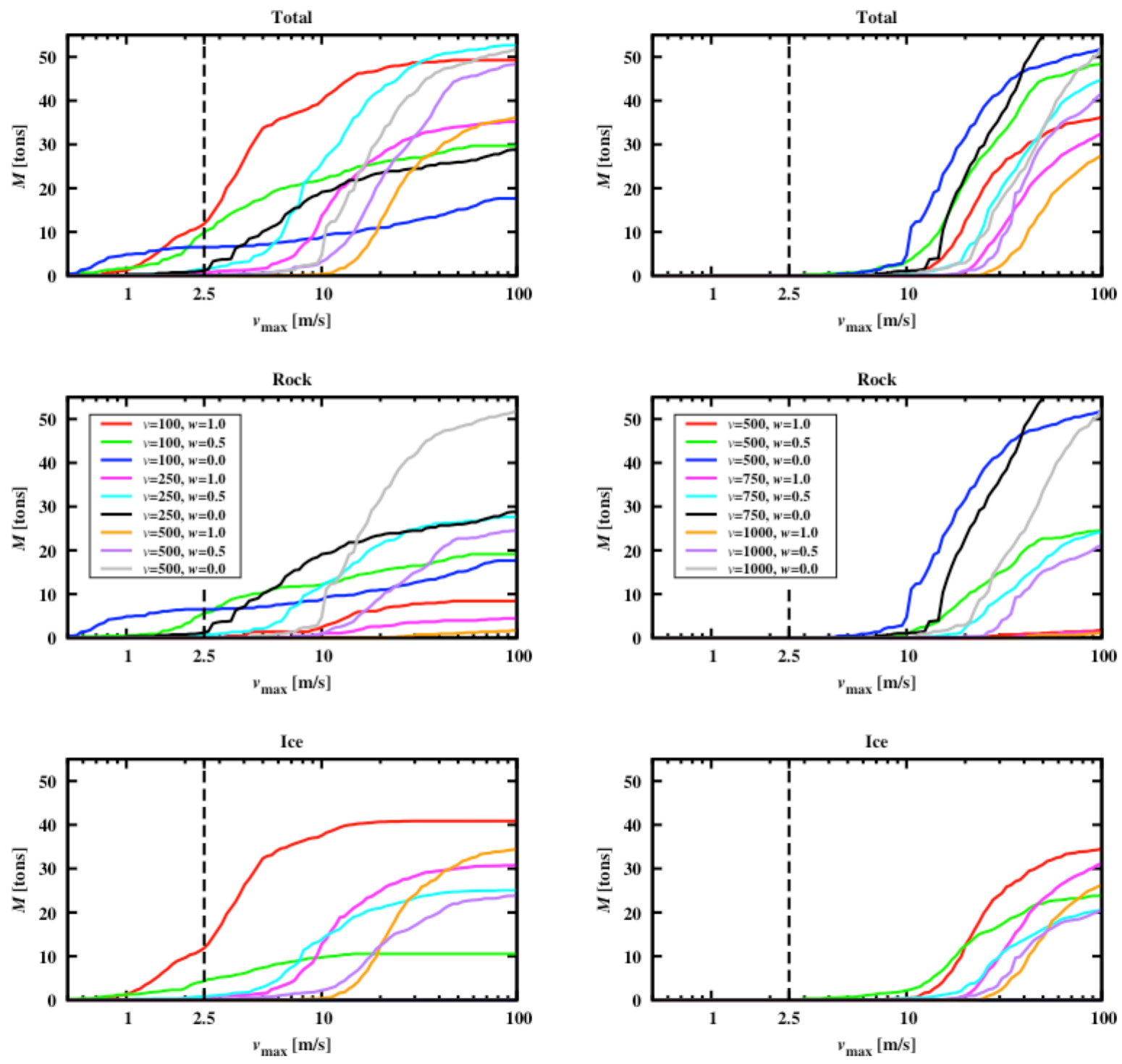

Fig. 8.- Graph of the ejected mass $(M)$ in terms of the maximum ejection velocity $\left(v_{\max }\right)$ for low, intermediate, and high impact velocities. The impactor is m-sized and the impact angle is $30^{\circ}$. The target is made of basalt with water-mass fraction of $w=0$ or 0.5 , or is pure ice with $w=1.0$. The dashed line at $2.5 \mathrm{~m} \mathrm{~s}^{-1}$ represents the upper limit of escape velocities of the currently known MBCs. 


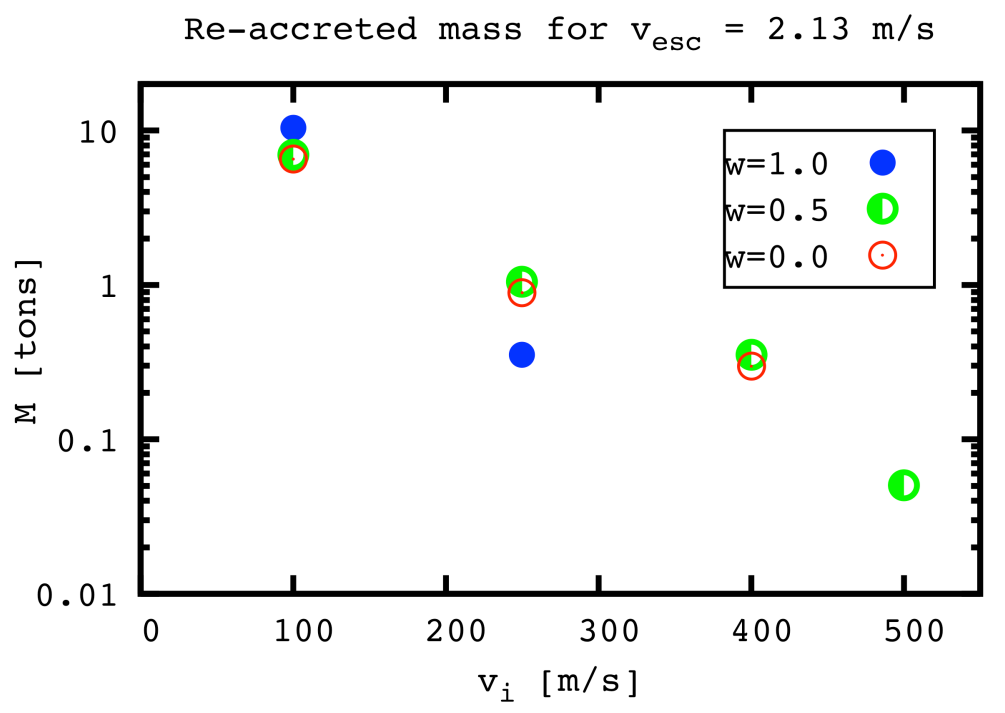

Fig. 9.- Re-accreted mass by a hypothetical MBC with the same size as that of $133 \mathrm{P} /(7968)$ Elst-Pizarro $\left(v_{\text {esc }}=2.13 \mathrm{~m} \mathrm{~s}^{-1}\right)$ but made of basalt in terms of the target water content $w$ and impact velocity $v_{\mathrm{i}}$ at a $30^{\circ}$ angle. 
Table 1. Physical properties of the currently known MBCs. The quantity $D_{\text {e }}$ represents an MBC's effective diameter, $a, e$ and $i$ are its semimajor axis, eccentricity and orbital inclination, respectively, $T_{\mathrm{J}}$ is its Tisserand number with respect to Jupiter, and $v_{\mathrm{esc}}$ is the value of its escape velocity. Adopted from Jewitt, Hsieh \& Agarwal (2015).

\begin{tabular}{lcccccc}
\hline \hline \multicolumn{1}{c}{ Object } & $D_{\mathrm{e}}[\mathrm{km}]$ & $a[\mathrm{AU}]$ & $e$ & $i[\mathrm{deg}]$ & $T_{\mathrm{J}}$ & $v_{\text {esc }}[\mathrm{m} / \mathrm{s}]$ \\
\hline 133P/(7968) Elst-Pizarro & $3.8 \pm 0.6$ & 3.157 & 0.165 & 1.39 & 3.184 & 2.13 \\
176P/(118401)LINEAR & $4.0 \pm 0.4$ & 3.196 & 0.192 & 0.24 & 3.167 & 1.95 \\
238P/Read (P/2005 U1) & 0.8 & 3.165 & 0.253 & 1.27 & 3.152 & $\ldots$ \\
259P/Garradd (P/2008 R1) & $0.3 \pm 0.02$ & 2.726 & 0.342 & 15.90 & 3.216 & 0.62 \\
P/2010 R2 (La Sagra) & 1.1 & 3.099 & 0.154 & 21.39 & 3.098 & 0.49 \\
288P/(300163) 2006 VW 139 & 3 & 3.050 & 0.200 & 3.24 & 3.203 & $\ldots$ \\
P/2012 T1 (PANSTARRS) & 2.4 & 3.154 & 0.236 & 11.06 & 3.134 & $\ldots$ \\
313P/Gibbs (P/2014 S4) & 1.0 & 3.156 & 0.242 & 10.97 & 3.132 & 0.86 \\
\hline
\end{tabular}




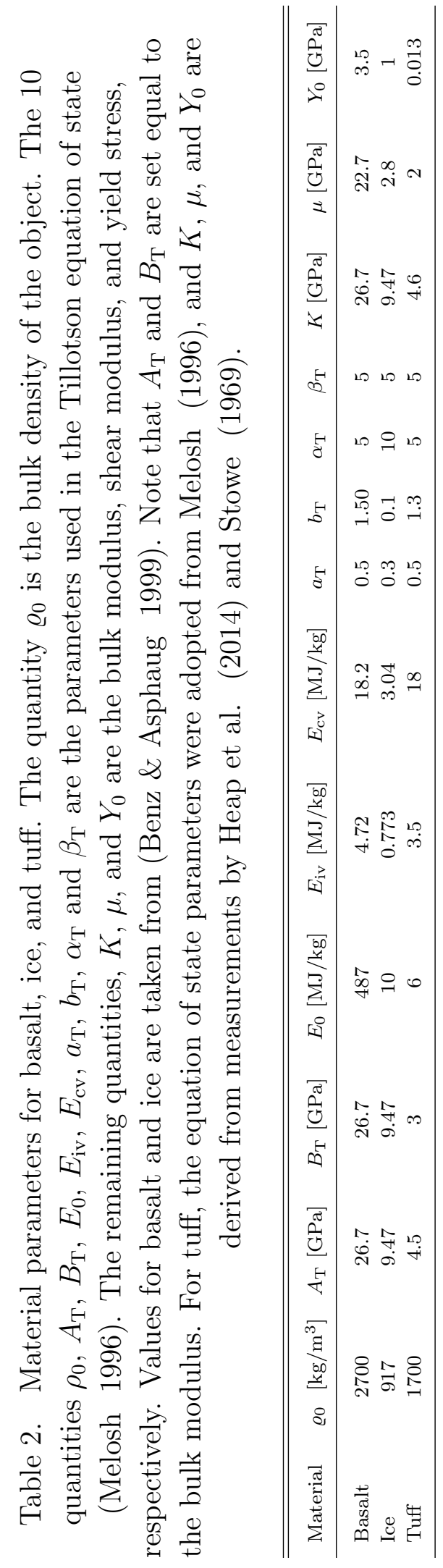


Table 3. The rate of the loss of water-ice at perihelion $\left(\dot{m}_{\mathrm{w}}\right)$ and the activation area $\left(A_{\text {act }}\right)$ of the currently known MBCs calculated using observational data from Hsieh (2014).

\begin{tabular}{lll}
\hline \hline \multicolumn{1}{c}{ Object } & $\dot{m}_{\mathrm{w}}\left[\mathrm{kg} \mathrm{s}^{-1}\right]$ & $A_{\text {act }}\left[\mathrm{m}^{2}\right]$ \\
\hline 133P/(7968) Elst-Pizarro & $7.2 \times 10^{-6}$ & $2 \times 10^{4}$ \\
176P/(118401) LINEAR & $8.3 \times 10^{-6}$ & $1 \times 10^{3}$ \\
238P/Read (P/2005 U1) & $1.3 \times 10^{-5}$ & $2 \times 10^{3}$ \\
259P/Garradd (P/2008 R1) & $3.4 \times 10^{-5}$ & - \\
288P/(300163) P/2006 VW 139 & $1.1 \times 10^{-5}$ & $5 \times 10^{3}$ \\
P/2010 R2 (La Sagra) & $7.6 \times 10^{-6}$ & $5 \times 10^{4}$ \\
P/2012 T1 (PANSTARRS) & $1.1 \times 10^{-5}$ & $1 \times 10^{4}$ \\
P/2013 R3 & $1.7 \times 10^{-5}$ & - \\
\hline
\end{tabular}

Note. - Hsieh et al. (2004) revised estimated $A_{\text {act }}$ to be a few tens to a few hundreds of $\mathrm{m}^{2}$. Hsieh et al. (2011) estimated $A_{\text {act }} \approx 2 \times 10^{4} \mathrm{~m}^{2}$ for $238 \mathrm{P} / \operatorname{Read}(\mathrm{P} / 2005 \mathrm{U} 1)$. 